\section{To zero or to hold control inputs with lossy links?}

\author{
Luca Schenato
}

\begin{abstract}
This paper studies the linear quadratic (LQ) performance of networked control systems where control packets are subject to loss. In particular we explore the two simplest compensation strategies commonly found in the literature: the zero-input strategy, in which the input to the plant is set to zero if a packet is dropped, and the holdinput strategy, in which the previous control input is used if packet is lost. We derive expressions for computing the optimal static gain for both strategies and we compare their performance on some numerical examples. Interestingly, none of the two can be claimed superior to the other, even for simple scalar systems, since there are scenarios where one strategy performs better then the other and scenarios where the converse occurs.
\end{abstract}

\section{INTRODUCTION}

Today's technological advances in wireless communications and in the fabrication of inexpensive embedded electronic devices, are creating a new paradigm where a large number of systems are interconnected, thus providing an unprecedented opportunity for totally new distributed control applications, commonly referred as networked control systems [1]. One of the most common problems in networked control systems, especially in wireless sensor networks, is packet drop, i.e. packets can be lost due to communication noise, interference, or congestion. If the controller is not co-located with the sensors and the actuators and it is placed in a remote location, then both sensor measurement packets and control packets can be lost.

A large number of works in the literature have analyzed estimation and filter design under lossy communication between the sensors and the controller [2][3][4][5][6][7][8][9]. However, there are also several works that studied the close loop performance when control packets can be dropped [4][10][11][12][13][14][15]. In general, in most of the literature two different strategies are considered for dealing with packet drops. In the first one, which we refer as zeroinput, the actuator input to the plant is set to zero when the control packet from the controller to the actuator is lost [12][13][14][15], while in the second, which we refer as hold-input, the latest control input stored in the actuator buffer is used when a packet is lost [10][4][11]. These are not the only strategies that can be adopted. In fact, if smart actuators are available, i.e. if actuators are provided with computational resources, then the whole controller [14] or a compensation filter [16] can be placed on the actuator. Another strategy is to use a model predictive controller which sends not only the current input but also a finite window of future control inputs into a single packet so that if a packet is lost the actuator can pop up from its buffer the corresponding predicted input from the latest received packet [17] [18]. Nonetheless, even this strategy requires more computational resources and communication bandwidth than the zero-input or hold-input strategies.

To the author's knowledge there is no study present in the literature which directly compares the hold-input and zero-input strategies, except for a simple empirical example in [14]. In particular, it seems that the zero-input strategy is mainly used for mathematical convenience as it gives simpler equations than the hold-input strategy, rather than being based on performance considerations. Indeed, intuitively one is led to think that the use the latest control input stored in the actuator buffer provides better performance than the use of a zero

This research is supported partially by CARIPARO foundation.

L. Schenato is with Faculty of Information Engineering, University of Padova, Via Gradenigo 6/b, 35131 Padova, Italy, schenato@dei.unipd.it input, in particular during the transient, since the true current optimal control input is likely to be close to the previous value. The zero-input strategy, however, it is not so unreasonable, since the optimal control input eventually converges to zero for a stable closed loop system in steady state. Motivated by these observations, the goal of this paper is to explicitly quantify the performance of these two strategies by adopting a linear quadratic (LQ) approach for discrete time linear system where the control input packets are dropped according to a Bernoulli stochastic process as described in details the Section II. In particular, we derive equations to compute the optimal static control gains for both strategies. While the equations for optimal control under the zero-input strategy in Section III have been previously derived [14], the equations for optimal gain design under the holdinput strategy presented in Section IV are novel. The equations are then used to compare the performance of the two strategies for scalar systems in Section V. In particular, we show that none of the two strategies is always superior to the other, but the performance depends on the packet loss probability and the cost weights. Finally, in Section VI we summarize the results and discuss future research directions.

\section{Problem Formulation}

Consider the following linear stochastic system:

$$
x_{k+1}=A x_{k}+B u_{k}^{a}
$$

where $u_{k}^{a} \in \mathbb{R}^{m}$ is the control input to the actuator, $A \in \mathbb{R}^{n \times n}$ and $B \in \mathbb{R}^{n \times m}$. We assume that the full state $x_{k} \in \mathbb{R}^{n}$ is available to a remote controller which adopts a simple linear feedback:

$$
u_{k}^{c}=L x_{k}
$$

where $L \in \mathbb{R}^{m \times n}$. The link between the controller and the actuator is lossy, and the stochastic binary variable $\nu_{k} \in\{0,1\}$ models the packet loss between the controller and the actuator. We consider two control strategies. In the zero-input strategy, if the packet is correctly delivered then $u_{k}^{a}=u_{k}^{c}$, otherwise the actuator does nothing, i.e. $u_{k}^{a}=0$, which gives the following closed loop system:

$$
\begin{aligned}
& x_{k+1}=A x_{k}+B u_{k}^{a} \\
& u_{k}^{a}=\nu_{k} u_{k}^{c} \\
& u_{k}^{c}=L_{z} x_{k}
\end{aligned}
$$

In the hold-input strategy, instead, when the packet is lost we use the previous control value stored in actuator, i.e. $u_{k}^{a}=u_{k-1}^{a}$, which leads to the following closed loop dynamics:

$$
\begin{aligned}
& x_{k+1}=A x_{k}+B u_{k}^{a} \\
& u_{k}^{a}=\nu_{k} u_{k}^{c}+\left(1-\nu_{k}\right) u_{k-1}^{a} \\
& u_{k}^{c}=L_{h} x_{k}
\end{aligned}
$$

The subscripts $z$ and $h$ in gains $L_{z}$ and $L_{h}$ are used to indicate the zero-input and the hold-input strategy, respectively. These two control packet loss compensation strategies are graphically illustrated in Figure 1.

We compare the performance in terms of the infinite horizon expected total cost:

$$
J_{\infty}(L)=\mathbb{E}\left[\sum_{k=0}^{\infty} x_{k}^{T} W x_{k}+\left(u_{k}^{a}\right)^{T} U u_{k}^{a}\right]
$$

where $W_{k} \in \mathbb{S}_{n}, U_{k} \in \mathbb{S}_{m}$, and $\mathbb{S}_{\ell}=\left\{S \in \mathbb{R}^{\ell \times \ell} \mid S=S^{T}, \xi^{T} S \xi \geq\right.$ $\left.0, \forall \xi \in \mathbb{R}^{\ell}\right\}$, i.e. $\mathbb{S}_{\ell}$ is the set of positive semidefinite matrices of dimension $\ell$. For simplicity we will often use the notation $X \geq 0$ as a shortcut for $X \in \mathbb{S}_{\ell}$. Note that only the inputs that actually enter the plant $u_{k}^{a}$, and not the desired control inputs $u_{k}^{c}$, are penalized. We also assume that the packet drops are i.i.d. Bernoulli random variables:

$$
\mathbb{P}\left[\nu_{k}=0\right]=\nu
$$



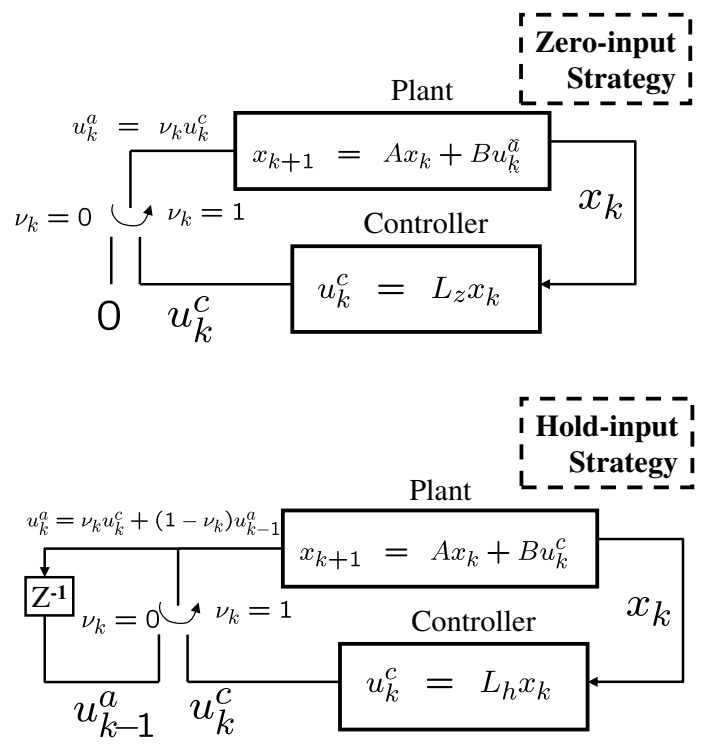

Fig. 1. Compensation approaches for actuators with no computational resources when a control packet is lost: zero-input approach $u_{k}^{a}=0$ (top) and hold-input approach $u_{k}^{a}=u_{k-1}^{a}$ (bottom).

where $\nu \in[0,1]$. More realistic packet loss models, which also include burst losses, can be captured with Markovian packet drops [15][4], however this approach leads to a more complex analysis and it is left as future work. A useful result that links stability to infinite horizon cost is the following:

Proposition 1: Let the pair $\left(A, W^{1 / 2}\right)$ be detectable, then the closed loop systems given by Equations (2) and (3) are mean square asymptotically stable, i.e. $\lim _{k \rightarrow \infty} \mathbb{E}\left[\left\|x_{k}\right\|^{2}\right]=0$, if and only if the cost defined in Equation (4) is bounded, i.e. $J_{\infty}(L)<\infty$.

Proof: The proof is rather standard since it can be derived similarly to standard LQ-control results [19], therefore it is omitted.

This proposition states that minimizing the infinite horizon cost implicitly solves the problem of finding a stabilizing gain $L$. In the next two sections, we will derive the optimal infinite horizon cost and corresponding optimal gain for the two strategies.

\section{LQ OPTIMAL CONTROL: ZERO-INPUT STRATEGY}

The equations in this sections have been previously derived in [14] in a more general LQG optimal control setting, but are reported here in the context of LQ control to ease comparison with the hold input strategy developed in the next section.

The optimal control equations are obtained using the standard dynamic programming approach, i.e. we compute the cost-to-go function iteratively. First note that system (2) can be written as

$$
\begin{aligned}
x_{k+1} & =\left(A+\nu_{k} B L\right) x_{k} \\
u_{k}^{a} & =\nu_{k} L x_{k}
\end{aligned}
$$

Let us define the cost-to-go function $C_{k}$ as follows

$$
C_{k}^{N}\left(x_{k}\right)=\mathbb{E}\left[\sum_{h=k}^{N} x_{h}^{T} W_{h} x_{h}+u_{h}^{a T} U_{h} u_{h}^{a} \mid x_{k}\right]
$$

where $W_{k}=W$ and $U_{k}=U$ except for the terminal costs $U_{N}=0$ and $W_{N} \geq 0$. We claim that the cost-to-go function can be written as

$$
C_{k}^{N}\left(x_{k}\right)=x_{k}^{T} S_{k} x_{k}
$$

where $S_{k} \in \mathbb{S}_{n}$. This is clearly true for $k=N$ with $S_{N}=W$. Then by induction, we show that this is true for all $k$. Suppose that it is true for $k+1$, then we have:

$$
\begin{aligned}
C_{k}^{N}\left(x_{k}\right)= & \mathbb{E}\left[\sum_{h=k}^{N} x_{h}^{T} W x_{h}+u_{h}^{a T} U u_{h}^{a} \mid x_{k}\right] \\
= & \mathbb{E}\left[x_{k}^{T} W x_{k}+u_{k}^{a T} U u_{k}^{a}+C_{k+1}^{N} \mid x_{k}\right] \\
= & \mathbb{E}\left[x_{k}^{T} W x_{k}+\nu_{k} x_{k}^{T} L^{T} U L x_{k}+\right. \\
& \left.+x_{k}^{T}\left(A+\nu_{k} B L\right)^{T} S_{k+1}\left(A+\nu_{k} B L\right) x_{k} \mid x_{k}\right] \\
= & x_{k}^{T}\left(W+\mathbb{E}\left[\nu_{k}\right] L^{T} U L+A S_{k+1} A^{T}+\right. \\
& \left.+2 \mathbb{E}\left[\nu_{k}\right] L^{T} B^{T} S_{k+1} A+\mathbb{E}\left[\nu_{k}^{2}\right] L^{T} B^{T} S_{k+1} B L\right) x_{k} \\
= & x_{k}^{T}\left(W+(1-\nu) L^{T} U L+\nu A^{T} S_{k+1} A+\right. \\
& \left.+(1-\nu)(A+B L)^{T} S_{k+1}(A+B L)\right) x_{k}
\end{aligned}
$$

where we used the fact that $\nu_{k}$ is independent of $x_{k}$, and $\mathbb{E}\left[\nu_{k}\right]=$ $\mathbb{E}\left[\nu_{k}^{2}\right]=1-\nu$. Therefore the claim above is true and the matrix $S_{k}$ is given by:

$$
\begin{aligned}
S_{k}= & W+\nu A^{T} S_{k+1} A+(1-\nu)\left(L^{T} U L+\right. \\
& \left.+(A+B L)^{T} S_{k+1}(A+B L)\right)=\mathcal{F}\left(S_{k+1}, L\right)
\end{aligned}
$$

where the operator $\mathcal{F}(S, L): \mathbb{S}_{n} \times \mathbb{R}^{n \times m} \rightarrow \mathbb{S}_{n}$ is affine in $S$ for fixed $L$, and quadratic in $L$ for fixed $S$. The infinite horizon cost can be obtained from the cost-to-go function as follows:

$$
J_{\infty}(L)=\lim _{N \rightarrow \infty} C_{0}^{N}\left(x_{0}\right)=x_{0}^{T} S x_{0}
$$

where $S$ is the positive semidefinite solution of the Lyapunov-like equation $S=\mathcal{F}(S, L)$, if such solution exists. The optimal gain $L^{*}$ is defined as the minimizer of the infinite horizon cost, i.e. $L^{*}=\operatorname{argmin}_{L} x_{0}^{T} S x_{0}$. It was shown in [14] that the optimal gain is independent of the initial condition $x_{0}$ and can be obtained by solving a Riccati-like equation. We summarize those results in the following theorem:

Theorem 1 ([14]): Consider the system defined by Equations (2) and the infinite horizon cost defined in Equation (4). Assume that the pair $(A, B)$ is stabilizable and $\left(A, W^{1 / 2}\right)$ is detectable. Then the optimal infinite horizon cost $J_{\infty}^{*}=\min _{L} J_{\infty}(L)$ is given by $J_{\infty}^{*}=x_{0} S^{*} x_{0}$ where $S^{*}$ is the unique positive semidefinite solution of the Riccati-like equation:

$$
\begin{aligned}
S^{*}= & A^{T} S^{*} A+W- \\
& -(1-\nu) A^{T} S^{*} B\left(B^{T} S^{*} B+U\right)^{\dagger} B^{T} S^{*} A=\Phi\left(S^{*}\right)
\end{aligned}
$$

and the optimal gain is given by

$$
L^{*}=-\left(B^{T} S^{*} B+U\right)^{\dagger} B^{T} S^{*} A
$$

where the symbol $\dagger$ indicates the pseudo-inverse operation. The Riccati-like equation $S_{\infty}^{*}=\Phi\left(S_{\infty}^{*}\right)$ has a positive semidefinite solution if and only if $\nu\left\langle\nu_{c}\right.$, where $\nu_{c}$ is a critical packet loss probability, which depends on the pair $(A, B)$. The critical loss probability $\nu_{c}$ satisfies the following bounds:

$$
\begin{aligned}
& \nu_{m} \leq \nu_{c} \leq \nu_{M} \\
& \nu_{M}=\frac{1}{\max _{i}\left|\lambda_{i}^{u}\right|^{2}}, \quad \nu_{m}=\frac{1}{\prod_{i}\left|\lambda_{i}^{u}\right|^{2}}
\end{aligned}
$$

where $\lambda_{i}^{u}$ are the unstable eigenvalues of the matrix $A$. In particular $\nu_{c}=\nu_{M}$ if $B$ is invertible, and $\nu_{c}=\nu_{m}$ if $B$ is rank one. 


\section{LQ OPTIMAL CONTROL: HOLD-INPUT STRATEGY}

We now derive the equations to compute the infinite horizon cost for the hold-input strategy. We proceed similarly to the previous section by computing the cost-to-go function. We first define the augmented state $z_{k}=\left[\begin{array}{c}x_{k} \\ u_{k-1}^{a}\end{array}\right] \in \mathbb{R}^{n+m}$. Then the system defined by Equations (3) can be written as:

$\left[\begin{array}{c}x_{k+1} \\ u_{k}^{a}\end{array}\right]=\left[\begin{array}{cc}A+\nu_{k} B L & \left(1-\nu_{k}\right) B \\ \nu_{k} L & \left(1-\nu_{k}\right) I\end{array}\right]\left[\begin{array}{c}x_{k} \\ u_{k-1}^{a}\end{array}\right]=F\left(\nu_{k}\right) z_{k}$

where $I \in \mathbb{R}^{m \times m}$ is the identity matrix. The evolution of the systems can be modeled as a Jump Markov Linear System (JMLS) since the dynamics jumps between two systems $F\left(\nu_{k}=1\right)$ and $F\left(\nu_{k}=\right.$ 0 ) according to a Bernoulli distribution. Many mathematical tools exist to study JMLS by reframing the LQ-control problem as the solution of a set of coupled algebraic Riccati equations (CARE) [20]. However, since the system we are considering has only two jumping dynamics with a simple Bernoulli switching, we can explicitly reduce it to a single Riccati-like equation that can be compared with the zeroinput Riccati-like equation of the previous section. We start defining the cost-to-go function as in the previous section:

$$
C_{k}^{N}\left(z_{k}\right)=\mathbb{E}\left[\sum_{h=k}^{N} x_{k}^{T} W_{k} x_{k}+u_{k}^{a T} U_{k} u_{k}^{a} \mid z_{k}\right]
$$

where $W_{k}=W$ and $U_{k}=U$ except for the terminal cost $U_{N}=0$ and $W_{N} \geq 0$. We claim that the cost-to-go function can be written as

$$
C_{k}^{N}\left(z_{k}\right)=z_{k}^{T} V_{k} z_{k}
$$

where $V_{k} \in \mathbb{S}_{n+m}$. This is clearly true for $k=N$ with $V_{N}=$ $\left[\begin{array}{cc}W_{N} & 0 \\ 0 & 0\end{array}\right]$. Then by induction, we show this is true for all $k$. Suppose it is true for $k+1$, then we have:

$$
\begin{aligned}
C_{k}^{N}\left(z_{k}\right)= & \mathbb{E}\left[\sum_{h=k}^{N} x_{k}^{T} W x_{k}+u_{k}^{a T} U u_{k}^{a} \mid z_{k}\right] \\
= & \mathbb{E}\left[x_{k}^{T} W x_{k}+u_{k}^{a T} U u_{k}^{a}+C_{k+1}^{N} \mid x_{k}\right] \\
= & \mathbb{E}\left[z_{k}^{T}\left[\begin{array}{cc}
W+\nu_{k} L^{T} U L & \nu_{k}\left(1-\nu_{k}\right) L^{T} U \\
\nu_{k}\left(1-\nu_{k}\right) U L & \left(1-\nu_{k}\right)^{2} U
\end{array}\right] z_{k}+\right. \\
& \left.+z_{k}^{T} F^{T}\left(\nu_{k}\right) V_{k+1} F\left(\nu_{k}\right) z_{k} \mid z_{k}\right] \\
= & z_{k}^{T}\left(\left[\begin{array}{cc}
W+\mathbb{E}\left[\nu_{k}\right] L^{T} U L & \mathbb{E}\left[\nu_{k}\left(1-\nu_{k}\right)\right] L^{T} U \\
\mathbb{E}\left[\nu_{k}\left(1-\nu_{k}\right)\right] U L & \mathbb{E}\left[\left(1-\nu_{k}\right)^{2}\right] U
\end{array}\right]+\right. \\
+ & \left.\mathbb{P}\left[\nu_{k}=0\right] F^{T}(0) V_{k+1} F(0)+\mathbb{P}\left[\nu_{k}=1\right] F^{T}(1) V_{k+1} F(1)\right) z_{k} \\
= & z_{k}^{T}\left(\left[\begin{array}{cc}
W+(1-\nu) L^{T} U L & 0 \\
0 & \nu U
\end{array}\right]+\right. \\
& \left.+\nu F^{T}(0) V_{k+1} F(0)+(1-\nu) F^{T}(1) V_{k+1} F(1)\right) z_{k}
\end{aligned}
$$

where we used the fact that $\nu_{k}$ is independent of $z_{k}$. Therefore the claim above is true and the matrix $V_{k}$ is given by:

$$
\begin{aligned}
V_{k}= & {\left[\begin{array}{cc}
W+(1-\nu) L^{T} U L & 0 \\
0 & \nu U
\end{array}\right]+} \\
& +\nu\left[\begin{array}{cc}
A^{T} & 0 \\
B^{T} & I
\end{array}\right] V_{k+1}\left[\begin{array}{cc}
A & B \\
0 & I
\end{array}\right]+ \\
& +(1-\nu)\left[\begin{array}{cc}
(A+B L)^{T} & L^{T} \\
0 & 0
\end{array}\right] V_{k+1}\left[\begin{array}{cc}
A+B L & 0 \\
L & 0
\end{array}\right] \\
= & \mathcal{L}_{\nu}\left(V_{k+1}, L\right)
\end{aligned}
$$

where the operator $\mathcal{L}_{\nu}(V, L): \mathbb{S}_{n+m} \times \mathbb{R}^{n \times m} \rightarrow \mathbb{S}_{n+m}$ is affine in $V$ for fixed $L$, and quadratic in $L$ for fixed $V$, and we made explicit its dependence on the parameter $\nu$ with the subscript. The property $\mathcal{L}_{\nu}(V, L) \geq 0$ if $V \geq 0$ follows by observing that all terms in the previous equation are of the form $X^{T} Y X$ with $Y \geq 0$ for proper choices of $X$ and $Y$, i.e. they are all positive semidefinite since $\xi^{T} X^{T} Y X \xi=\left\|Y^{\frac{1}{2}} X \xi\right\| \geq 0$. Let us partition the matrix $V$ and the operator $\mathcal{L}_{\nu}(V, L)$ as follows

$$
V=\left[\begin{array}{ll}
V_{11} & V_{12} \\
V_{12}^{T} & V_{22}
\end{array}\right], \quad \mathcal{L}_{\nu}=\left[\begin{array}{cc}
\mathcal{L}_{11} & \mathcal{L}_{12} \\
\mathcal{L}_{12}^{T} & \mathcal{L}_{22}
\end{array}\right]
$$

where $V_{11} \in \mathbb{S}_{n}, V_{12} \in \mathbb{R}^{n \times m}, V_{22} \in \mathbb{S}_{m}$, then Equation (15) can be written as:

$$
\begin{aligned}
\mathcal{L}_{11}(V, L)= & W+\nu A^{T} V_{11} A+(1-\nu)\left(L^{T} U L+\right. \\
& +L^{T} V_{22} L+(A+B L)^{T} V_{11}(A+B L)+ \\
& \left.+L^{T} V_{12}^{T}(A+B L)+(A+B L)^{T} V_{12} L\right) \\
\mathcal{L}_{12}(V)= & \nu\left(A^{T} V_{11} B+A^{T} V_{12}\right) \\
\mathcal{L}_{22}(V)= & \nu\left(U+B^{T} V_{11} B+V_{12}^{T} B+B V_{12}+V_{22}\right)
\end{aligned}
$$

$\mathcal{L}_{11}(V, L): \mathbb{S}_{n+m} \times \mathbb{R}^{n \times m} \rightarrow \mathbb{S}_{n}, \mathcal{L}_{12}(V): \mathbb{S}_{n+m} \rightarrow$ $\mathbb{R}^{n \times m}, \mathcal{L}_{22}(V): \mathbb{S}_{n+m} \rightarrow \mathbb{S}_{m}$. Note that only the upper left block of $\mathcal{L}_{\nu}(V, L)$, i.e. $\mathcal{L}_{11}(V, L)$, depends on the gain $L$, and that $\mathcal{L}_{\nu}(V, L) \geq 0$ implies also $\mathcal{L}_{11}(V, L) \geq 0$ and $\mathcal{L}_{22}(V) \geq 0$. Moreover, since it is quadratic in the gain $L$ it can be written as follows:

$$
\begin{aligned}
\mathcal{L}_{11}(L, V)= & \Phi_{\nu}(V)+(1-\nu)\left(L-L_{V}\right)^{T} P_{V}\left(L-L_{V}\right) \\
\Phi_{\nu}(V)= & W+A^{T} V_{11} A- \\
& -(1-\nu) A^{T}\left(V_{11} B+V_{12}\right) P_{V}^{\dagger}\left(B^{T} V_{11}+V_{12}^{T}\right) A \\
P_{V}= & U+V_{22}+B^{T} V_{11} B+V_{12}^{T} B+B^{T} V_{12} \\
L_{V}= & -P_{V}^{\dagger}\left(B^{T} V_{11}+V_{12}^{T}\right) A
\end{aligned}
$$

We define the nonlinear operator $\Psi_{\nu}(V): \mathbb{S}_{n+m} \rightarrow \mathbb{S}_{n+m}$ as follows:

$$
\Psi_{\nu}(V)=\left[\begin{array}{cc}
\Phi_{\nu}(V) & \mathcal{L}_{12}(V) \\
\mathcal{L}_{12}^{T}(V) & \mathcal{L}_{22}(V)
\end{array}\right]
$$

which has few useful properties summarized in the following proposition:

Proposition 2: Consider the operators $\mathcal{L}_{\nu}(L, V)$ and $\Psi_{\nu}(V)$ defined in Equations (15) and (23), respectively. Also assume that $V \geq 0$. Then the following facts are true:

(a) $\mathcal{L}_{\nu}(L, V) \geq \Psi_{\nu}(V)=\mathcal{L}_{\nu}\left(L_{V}, V\right) \geq 0, \forall L, V$, where $L_{V}$ is defined in Equation (22).

(b) If $V_{1} \geq V_{2}$ then $\mathcal{L}_{\nu}\left(L, V_{1}\right) \geq \mathcal{L}_{\nu}\left(L, V_{2}\right)$ and $\Psi_{\nu}\left(V_{1}\right) \geq$ $\Psi_{\nu}\left(V_{2}\right)$.

(c) If $\nu_{1} \geq \nu_{2}$ then $\Psi_{\nu_{1}}(V) \geq \Psi_{\nu_{2}}(V), \forall V$.

(d) If $\left(A, W^{1 / 2}\right)$ is detectable and $V=\Psi_{\nu}(V)$ and $S=$ $\mathcal{L}_{\nu}(L, S)$ have a positive semidefinite solution, then $V$ and $S$ are unique, $V$ is stabilizing, and $V \leq S, \forall S$. Moreover, if $L=L_{V}$, then $V=S$.

Proof: Note that the identity $\Psi_{\nu}(V)=\mathcal{L}_{\nu}\left(L_{V}, V\right)$ can be checked by direct substitution of $L_{V}$ into the operator $\mathcal{L}_{\nu}$. Fact (a) follows from:

$\mathcal{L}_{\nu}(L, V)-\Psi_{\nu}(V)=\left[\begin{array}{cc}(1-\nu)\left(L-L_{V}\right)^{T} P_{V}\left(L-L_{V}\right) & 0 \\ 0 & 0\end{array}\right] \geq 0$

for all $L$ and $V$, where $P_{V}$ is defined above.

In fact (b) the monotonicity of $\mathcal{L}_{\nu}$ follows from $\mathcal{L}_{\nu}\left(L, V_{1}\right)-$ $\mathcal{L}_{\nu}\left(L, V_{2}\right)=\left.\mathcal{L}_{\nu}\left(L, V_{1}-V_{2}\right)\right|_{U=0, W=0} \geq 0$. The monotonicity of $\Psi_{\nu}$ follows from fact (a) since $\Psi_{\nu}\left(V_{1}\right)=\mathcal{L}_{\nu}\left(L_{V_{1}}, V_{1}\right) \geq$ $\mathcal{L}_{\nu}\left(L_{V_{1}}, V_{2}\right) \geq \Psi_{\nu}\left(V_{2}\right)$.

Fact (c) follows from:

$$
\Psi_{\nu_{1}}(V)-\Psi_{\nu_{2}}(V)=\left(\nu_{1}-\nu_{2}\right)\left[\begin{array}{cc}
X P_{V}^{\dagger} X^{T} & X \\
X^{T} & P_{V}
\end{array}\right] \geq 0, \forall V
$$


where $X=A^{T}\left(V_{11} B+V_{12}\right)$, since the positive semidefiniteness can be verified by taking the Shur complement and noting that $P_{V} \geq 0$.

The proof for fact (d) is somewhat more technical and in the interest of space only a sketch is reported. The proof follows along the same lines of optimal estimation [19] [8], being optimal control simply its dual, where it is shown that the null space of the matrices $V$ and $S$ corresponds to the unobservable subspace of $\left(A, W^{1 / 2}\right)$, which, by hypothesis, is strictly stable. This property is sufficient to prove uniqueness of the solution, and that $V$ leads to a stabilizing gain $L_{V}$. The inequality $V \leq S, \forall L$ follows directly from fact (a). The last statement $V=S$ if $L=L_{V}$ comes from fact (a) and the uniqueness of the solution for both operators $\mathcal{L}_{\nu}$ and $\Psi_{\nu}$.

We are now ready to derive the optimal gain and stability conditions for the hold-input strategy:

Theorem 2: Consider the system defined by Equations (3) and the infinite horizon cost defined in Equation (4). Assume that the pair $(A, B)$ is stabilizable and $\left(A, W^{1 / 2}\right)$ is detectable. Then optimal cost is given by

$$
J_{\infty}^{*}=\min _{L} J_{\infty}(L)=z_{0}^{T} V^{*} z_{0}
$$

where $V^{*}$ is the unique stabilizing positive semidefinite solution of the Riccati-like equation:

$$
V^{*}=\Psi_{\nu}\left(V^{*}\right)
$$

where $\Psi_{\nu}(V)$ is defined in Equation (23) and the corresponding optimal gain is given by

$$
L^{*}=L_{V^{*}}
$$

where $L_{V}$ is defined in Equation (22). The Riccati-like equation $V^{*}=\Psi_{\nu}\left(V^{*}\right)$ has a unique stabilizing positive semidefinite solution if and only if $\nu<\nu_{c}$, where $\nu_{c}$ is a critical packet loss probability, which depends on the pair $(A, B)$ and satisfies the bound $\nu_{c} \geq \nu_{M}$, where $\nu_{M}$ is defined in Theorem 1. If $B$ is invertible then $\nu_{c}=\nu_{m}$. The fixed point $V^{*}$ can be obtained as the limit of the sequence $V_{k+1}^{*}=\Psi_{\nu}\left(V_{k}^{*}\right), V_{0}^{*}=0$, i.e. $\lim _{k \rightarrow \infty} V_{k}^{*}=V^{*}$. If $u_{-1}^{a}=0$, then the optimal cost reduces to $J_{\infty}^{*}=x_{0}^{T} V_{11}^{*} x_{0}$.

Proof: A detailed proof is rather technical and long but can be obtained by using similar tools as those presented in [8] and [14]. Therefore in the interest of space, only a sketch of the main steps of the proof are given. First note that it is sufficient to understand when $V^{*}=\Phi_{\nu}\left(V^{*}\right)$ has a solution, since fact (d) of of Proposition 2 guarantees uniqueness and stabilizability of the solution $V^{*}$. We start by noting that from the definition of cost-to-go function we have that $J_{\infty}(L)=\lim _{N \rightarrow \infty} C_{0}^{N}\left(z_{0}\right)=\lim _{N \rightarrow \infty} z_{0}^{T} \mathcal{L}_{\nu}^{N}\left(L, V_{N}\right) z_{0}$, where $\mathcal{L}_{\nu}^{k+1}(L, V)=\mathcal{L}_{\nu} \circ \mathcal{L}_{\nu}^{k}(L, V)$, since $V_{0}=\mathcal{L}_{\nu}^{N}\left(L, V_{N}\right)$. If we define $\widetilde{V}_{k}=\mathcal{L}_{\nu}^{k}\left(\widetilde{V}_{0}, L\right)$ where $\widetilde{V}_{0}=V_{N}$, then $J_{\infty}(L)=\lim _{N \rightarrow \infty} z_{0}^{T} \widetilde{V}_{N} z_{0}$. Let also consider the sequence $V_{k+1}^{*}=\Psi_{\nu}\left(V_{k}^{*}\right)$ where $V_{0}^{*}=V_{N}$. Note that $V_{N}$ becomes the initial condition for the two sequences.

The existence of the optimal cost can be inferred from the convergence conditions of the sequence $V_{k}^{*}$. Indeed, we can restrict our analysis to the convergence of $V_{k}^{*}$ and $\widetilde{V}_{k}$ from initial condition $V_{N}=0$, since uniqueness of fixed points of operators $\Phi_{\nu}$ and $\mathcal{L}_{\nu}$ implies convergence from any initial condition. From monotonicity of operators $\Phi_{\nu}(V)$ and $\mathcal{L}_{\nu}(V, L)$ with respect to $V$ (fact (b) of Proposition 2) and minimality of operator $\Phi_{\nu}(V)$ (fact (a) of Proposition 2), it follow that $V_{k+1}^{*} \geq V_{k}^{*}, \widetilde{V}_{k+1} \geq \widetilde{V}_{k}$ and $V^{*} \leq \widetilde{V}_{k}$, therefore the sequences either converge or are unbounded. If they converge then i.e. $\lim _{\tilde{\sigma}} \rightarrow \widetilde{V}_{k}=\widetilde{V}$ and $\lim _{k \rightarrow} V_{k}^{*}=V^{*}$, then they also need to satisfy $\widetilde{V}=\mathcal{L}_{\nu}(\widetilde{V}, L), V^{*}=\Psi_{\nu}\left(V^{*}\right)$ because of continuity of the operators in their arguments. If the system is mean square stabilizable, then there exists $L$ such that $\widetilde{V}$ exists, therefore all elements of the sequence $V_{k}^{*} \leq \widetilde{V}$ are bounded, consequently $V^{*}$ exists. Conversely, if $V^{*}$ exists, since it must necessarily be a stabilizing solution and unique, then $\lim _{N \rightarrow \infty} \mathcal{L}^{N}\left(V_{N}, L^{*}\right)=V^{*}$ for any $V_{N} \geq 0$.

Now we want to show that there exists $\nu_{c}$, such that $V_{\nu}^{*}=$ $\Psi_{\nu}\left(V_{\nu}^{*}\right) \geq 0$ has a solution if and only if $\nu<\nu_{c}$. In fact, by monotonicity of $\Psi_{\nu}$ in the parameter $\nu$ (fact (c) of Proposition 2), we necessarily have $V_{\nu_{1}}^{*} \leq V_{\nu_{2}}^{*}$ if $\nu_{1} \leq \nu_{2}$, therefore if $V_{\nu_{2}}^{*}$ exists, then also $V_{\nu_{1}}^{*}$ exists, and conversely if $V_{\nu_{1}}^{*}$ does not exist, then also $V_{\nu_{2}}^{*}$ does not exist. These two observations prove the existence of $\nu_{c}$.

We can also provide some additional considerations about $\nu_{c}$. First, let us consider the linear operator $\mathcal{G}_{\nu}$ associated to the affine operator $\mathcal{L}_{\nu}$ defined as $\mathcal{G}_{\nu}(V, L)=\mathcal{L}_{\nu}(V, L)-\mathcal{L}_{\nu}(0, L): \mathbb{S}_{n+m} \times \mathbb{R}^{n \times m} \rightarrow$ $\mathbb{S}_{n+m}$. If there exists $L$ such that $\mathcal{G}_{\nu}(\cdot, L)$ is strictly stable, then $V^{*}=\Psi_{\nu}\left(V^{*}\right)$ exists, and conversely if $V^{*}=\Psi_{\nu}\left(V^{*}\right)$, then $\mathcal{G}_{\nu}\left(\cdot, L_{V^{*}}\right)$ is strictly stable, since $V^{*}$ is unique and stabilizing. We start by showing that $\nu_{c}>0$. If $\nu=0$, i.e. if there is no packet loss, then we recover the standard LQ problem. In fact, $V^{*}=\left[\begin{array}{cc}V_{A R E} & 0 \\ 0 & 0\end{array}\right]$, where $V_{A R E}$ is the solution of the standard algebraic Riccati equation (ARE), i.e. $V_{A R E}=\Phi_{0}\left(V_{A R E}\right)$, and $L^{*}=L_{A R E}$ is the corresponding gain. Since $(A, B)$ is stabilizable and $\left(A, Q^{1 / 2}\right)$ is detectable, then the solution $V^{*}$ exists, is unique and it is a stabilizing solution [19], i.e. $\mathcal{G}\left(\cdot, L_{A R E}\right)$ is strictly stable and $\lim _{N \rightarrow \infty} \mathcal{L}_{\nu}^{N}\left(V_{N}, L_{A R E}\right)=V^{*}$ for any $V_{N} \geq 0$. The operators $\mathcal{L}_{\nu}, \mathcal{G}, \Psi_{\nu}$, as well the fixed point $V^{*}$ and optimal gain $L^{*}=L_{V^{*}}$, are all continuous in $\nu$, therefore, since $V^{*}$ leads to a stabilizing solution for $\nu=0$, i.e. the eigenvalues of the operator $\mathcal{G}\left(\cdot, L^{*}\right)$ are all strictly inside the unit circle, then by continuity $V^{*}=\Psi_{\nu}\left(V^{*}\right)$ exists, is unique and remains to be stabilizing if $\nu<\bar{\nu}$ for some $\bar{\nu}>0$. Since $V^{*}=\Psi_{\nu}\left(V^{*}\right)$ exists if and only if $\nu<\nu_{c}$, as we showed above, then $\bar{\nu}$ can be as large as $\nu_{c}$.

Finally, we show that $\nu_{c} \leq \nu_{M}=\frac{1}{\max _{i}\left|\lambda_{i}\right|^{2}}<1$ if $A$ is unstable. Let us define the following linear operator $\overline{\mathcal{L}}_{\nu}(V)=$ $\left[\begin{array}{ll}W+\nu A^{T} V_{11} A & \mathcal{L}_{12}(V)\end{array}\right]$ $\left.\mathcal{L}_{12}^{T}(V) \quad \mathcal{L}_{22}(V)\right]: \mathbb{S}_{n+m} \rightarrow \mathbb{S}_{n+m}$, where $V_{11}$ and the operators $\mathcal{L}_{12}(V), \mathcal{L}_{22}(V)$ were defined in Eqn. (16)-(18). Note that this operator is affine in $V$ and it has a fixed point $\bar{V}=$ $\overline{\mathcal{L}}_{\nu}(\bar{V}) \geq 0$ only if $\bar{V}_{11}=W+\nu A^{T} \bar{V}_{11} A \geq 0$, i.e. only if $\sqrt{\nu} A$ is strictly stable since $\bar{V}_{11}$ is the solution of a standard Lyapunov equation. In other words, $\bar{V}$ exists only if $\nu<\frac{1}{\max _{i}\left|\lambda_{i}\right|^{2}}$ where $\lambda_{i}$ are the eigenvalues of $A$. Since by direct inspection we observe that $\overline{\mathcal{L}}_{\nu}(V) \leq \Phi_{\nu}(V), \forall V \geq 0$, it follows that $\bar{V} \leq V^{*}$ by using usual monotonicity arguments. As a consequence we necessarily have $\nu_{c} \leq$ $\nu_{M}=\frac{1}{\max _{i}\left|\lambda_{i}\right|^{2}}$ if $A$ is unstable. We now show that $\nu_{c}=\nu_{M}$ if $\bar{B}$ is invertible. In this scenario let us consider $L=-B^{-1} A$, therefore $\mathcal{L}_{11}\left(V,-B^{-1} A\right)=W+\nu A^{T} V_{11} A$ and consequently the existence of $\tilde{V}=\mathcal{L}_{\nu}\left(\tilde{V},-B^{-1} A\right) \geq 0$ is equivalent to the existence of a fixed point for $V=\overline{\mathcal{L}}_{\nu}(V)$. Since $\Phi_{\nu}(V) \leq \mathcal{L}_{\nu}\left(V,-B^{-1} A\right), \forall V \geq 0$, then $\nu_{c} \geq \nu_{M}$, from which follows $\nu_{c}=\nu_{M}$. If $A$ is strictly stable then, it is easy to see that $\widetilde{V}=\mathcal{L}_{\nu}(\widetilde{V}, 0) \geq 0$ exists if and only if $\nu>0$. Noting $\Psi_{\nu=1}(V)=\mathcal{L}_{\nu=1}(\widetilde{V}, 0), \forall V$ and $\Psi_{\nu}(V) \leq \mathcal{L}_{\nu}(\widetilde{V}, 0), \forall V, \nu>0$, since $\widetilde{V}=\mathcal{L}_{\nu}(\widetilde{V}, 0)$ has a solution if and only if $\nu>0$, then by monotonicity arguments we can claim that $\nu_{c}=0$.

Note that the hypothesis $u_{-1}^{a}=0$ is a natural choice which allows a fair comparison between the zero-input strategy and the holdinput strategy. Few remarks are in order. The first remark is that the previous theorem states that we can compute the optimal gain $L^{*}$ and the corresponding optimal cost $J_{\infty}^{*}$ as the solution of a Riccati-like equation, which can be computed numerically through an iterative procedure. The second remark is that if the system $A$ is unstable, then there is a critical loss probability $\nu_{c}$ above which the closed loop system cannot be stabilized by any linear feedback. In general, it is hard to find the value for $\nu_{c}$ in closed form. However, using 
a bisection method based on whether the solution $V^{*}=\Psi_{\nu}\left(V^{*}\right)$ exists, we can numerically compute it with any desired accuracy. The last remark is that the optimal gain $L^{*}$ does not depend on the initial conditions $\left(x_{0}, u_{-1}^{a}\right)$. Although this is clear for classic LQ control and for the zero-input strategy, it is not trivial from Equation (15) that the matrix $V$ for hold-input strategy is convex in the gain $L$, since a multivariate quadratic function is not necessarily convex.

So far we have shown how to compute the optimal gain for both the zero-input strategy and for hold-input strategy. However, we have not yet determined whether one strategy is better than the other. In the next section, we will compare the performance of the two strategies for scalar systems, for which we can find closed form expressions for the gain $L$ and the performance $J_{\infty}$.

\section{HOLD-INPUT VS ZERO-INPUT: THE SCALAR CASE}

Without loss of generality, we assume that $B=1, A=a, W=w$, and $x_{0}=1$. Since $B$ is trivially invertible, if $A$ is unstable, then according to Theorem 1 and Theorem 2 the closed loop system under either the zero-input strategy or the hold-input strategy is stable if and only if $\nu<\nu_{M}=1 / a^{2}$. Therefore, both strategies have the same stability domain. Although they provide the same stabilizability, they might provide different performance, therefore in the following we compare them under different choices of the weights $W$ and $U$.

We start by assuming that $U=0$, which corresponds to the cheapcontrol scenario where we look for the fastest converging controller in mean square sense. In fact, for $\nu=0, U=0$ we obtain the usual dead-beat controller. If we substitute the systems parameters into Equations (9) and (10) for the zero-input strategy we get:

$$
\begin{aligned}
s^{*} & =w+a^{2} s^{*}-(1-\nu) a^{2} s^{*}=w+\nu a^{2} s^{*} \\
& =\frac{1}{1-\nu a^{2}} w \\
\ell_{z}^{*} & =-a
\end{aligned}
$$

Note that the optimal gain $\ell_{z}^{*}$ is independent of the packet loss rate $\nu$, which is very valuable since it implies that this gain is optimal also for unknown time-varying packet loss rates.

Similarly, if we substitute the system parameters into Equations (24) for the hold-input strategy we get:

$$
\begin{aligned}
v_{12}^{*} & =\nu a\left(v_{1}^{*}+v_{12}^{*}\right)=\frac{\nu a}{1-\nu a} v_{1}^{*} \\
v_{2}^{*} & =\nu\left(v_{1}^{*}+2 v_{12}^{*}+v_{2}^{*}\right)=\frac{\nu\left(v_{1}^{*}+2 v_{12}^{*}\right)}{1-\nu}=\frac{\nu(1+\nu a)}{(1-\nu)(1-\nu a)} v_{1}^{*} \\
v_{1}^{*} & =w+a^{2} v_{1}^{*}-(1-\nu) \frac{a^{2}\left(v_{1}^{*}+v_{12}^{*}\right)^{2}}{v_{1}^{*}+v_{2}^{*}+2 v_{12}^{*}}
\end{aligned}
$$

If we substitute the first two equations into the third one, we find the following expression for $v_{1}^{*}$ and the optimal gain $\ell_{h}^{*}$ given by Equation (25):

$$
\begin{aligned}
v_{1}^{*} & =w+a^{2} v_{1}^{*}-\frac{(1-\nu)^{2}}{1-\nu^{2} a^{2}} a^{2} v_{1}^{*} \\
& =\frac{\left(1-\nu^{2} a^{2}\right)}{\left(1-\nu a^{2}\right)^{2}} w \\
\ell_{h}^{*} & =-\frac{(1-\nu) a}{1+\nu a}
\end{aligned}
$$

We now show that the zero-input strategy gives a better performance than the hold-input strategy in the cheap-control scenario. If we compute the performance difference:

$s^{*}-v_{1}^{*}=\left(\frac{1}{1-\nu a^{2}}-\frac{1-\nu^{2} a^{2}}{\left(1-\nu a^{2}\right)^{2}}\right) w=\left(\frac{-(1-\nu) \nu a^{2}}{\left(1-\nu a^{2}\right)^{2}}\right) w \leq 0$, we see that it is always negative in the stability region $\nu<1 / a^{2}$, therefore the zero-hold strategy always outperforms the hold input strategy under the cheap-control scenario. Figure 2 shows a graphical representation of Equations (26) and (28), where $A=1.2, B=W=$ 1 , and $U=0$. In Figure 3 it is shown a typical realization for an

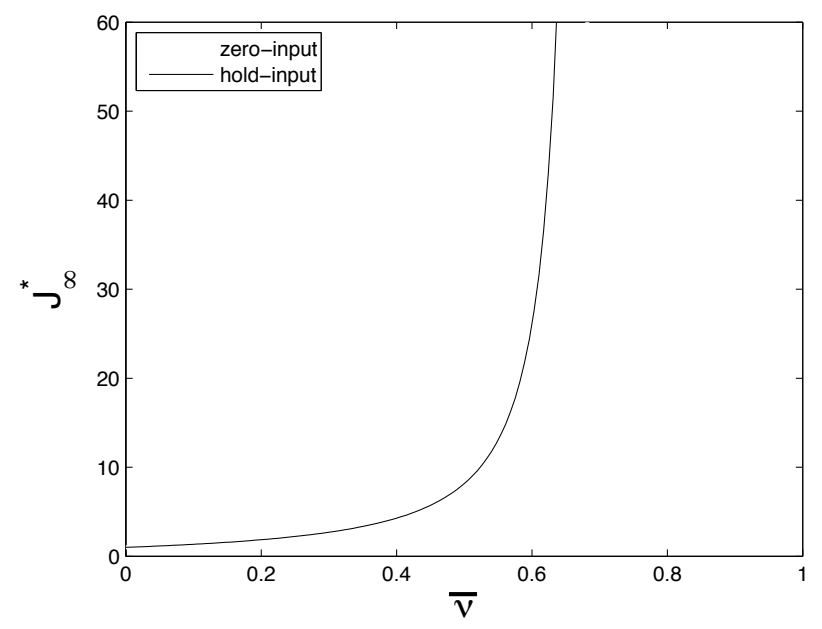

Fig. 2. Minimum cost $J_{\infty}$ for $A=1.2, B=W=x_{0}=1, U=0$ under zero-input and hold-input control architectures. The critical loss probability for this systems is $\nu_{c}=1 / 1.2^{2}=0.694$

unstable system, $A=1.2$, with packet loss probability $\nu=0.5$, using optimal gain $\ell_{z}^{*}=-a=-1.2$ for the zero-input strategy and $\ell_{h}^{*}=-(1-\nu) a /(1+\nu a)=-0.375$ for the hold-input strategy. Note that the first control packet is lost and the state $x$ starts to diverge, however as soon as a packet arrives the zero-hold strategy drives the system to zero, while the hold-input requires a longer time. The reason why the zero-input strategy performs better in the cheapinput scenario, is that if the hold-input strategy adopts the deadbeat gain, then as soon as a packet arrives the controller drives the system to zero, similarly to the zero-input strategy. However, if two or more packets are lost consecutively, the hold-input strategy will keep driving the system with a non-zero input, thus creating overshooting or oscillations.
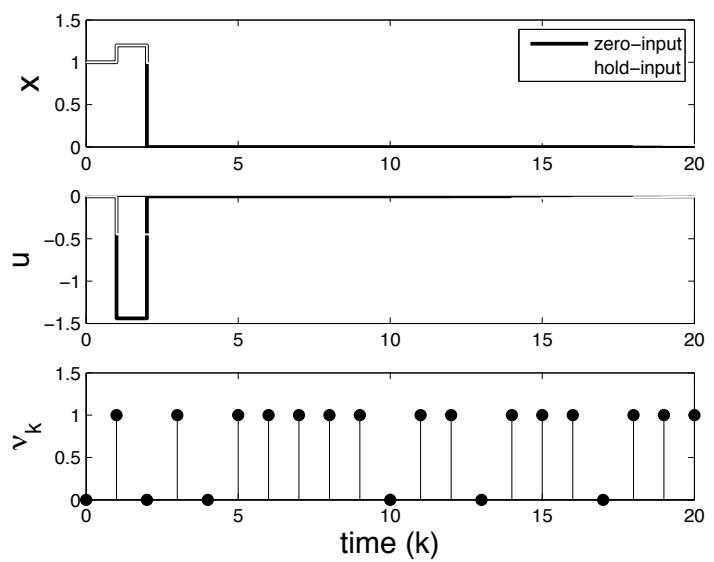

Fig. 3. A specific realization for $A=1.2, x_{0}=1, \nu=0.5$ under under optimal zero-input control, $\ell_{z}^{*}=-a=-1.2$ and optimal hold-input control, $\ell_{h}^{*}=-(1-\nu) a /(1+\nu a)=-0.375$.

So far we have considered only the case $U=0$, i.e. the case when the input it is not penalized. Unfortunately, if $U=u>0$, 
it is not possible to derive a closed form solution for the sets $\mathbb{D}_{z}=\left\{(\nu, u, w) \mid s^{*} \geq 0, v^{*} \geq 0, s^{*}<v^{*}\right\}$, i.e. the region where the zero-input strategy is superior than the hold-input strategy, and $\mathbb{D}_{h}=\left\{(\nu, u, w) \mid s^{*} \geq 0, v^{*} \geq 0, s^{*}>v^{*}\right\}$, i.e. the region where the opposite occurs. This is because both $s^{*}$ and $v^{*}$ are the positive solutions of second order equations, and in fact, after some straightforward algebraic manipulations their closed form expressions are given by:

$$
\begin{aligned}
s^{*} & =\frac{w+\left(a^{2}-1\right) u+\sqrt{\left(w+\left(a^{2}-1\right) u\right)^{2}+4 u w\left(1-\nu a^{2}\right)}}{2\left(1-\nu a^{2}\right)} \\
v^{*} & =\frac{\alpha+\sqrt{\left(\alpha^{2}+4\left(1-\nu a^{2}\right)^{4}(1-\nu)(1-\nu a)^{2} u w\right.}}{2\left(1-\nu a^{2}\right)^{2}} \\
\alpha & =w\left(1-\nu^{2} a^{2}\right)+(1-\nu)(1-\nu a)^{2}\left(a^{2}-1\right) u
\end{aligned}
$$

Nonetheless, we can numerically compute the optimality regions, which are displayed in Figure 4 for state cost $W=w=1$. Very interestingly, if the input penalization $u$ is sufficiently small, then the zero-input strategy is always better then the hold-input strategy for all packet loss values $\nu \in\left[0, \nu_{c}\right)$. Differently, if $u$ is sufficiently large, then for small packet loss probability rates $\nu$ the hold-input strategy outperforms the zero-input strategy, however for packet loss probability close to the critical one $\nu_{c}$, the zeroinput continues to be better. The reason why the hold-input strategy performs better for moderate packet loss rates and strong input penalization $U$ is that in this scenario the optimal input $u_{k}^{a}$ should be small in magnitude and slowly changing, i.e it decreases little from one time step to the next, differently from the dead-beat control input. Therefore, since the hold-input strategy provides a smoother sequence of inputs, it performs better. However, for large packet loss probability, long consecutive packet losses give rise again to overshooting and oscillations, thus providing a worse performance than the zero-input strategy.

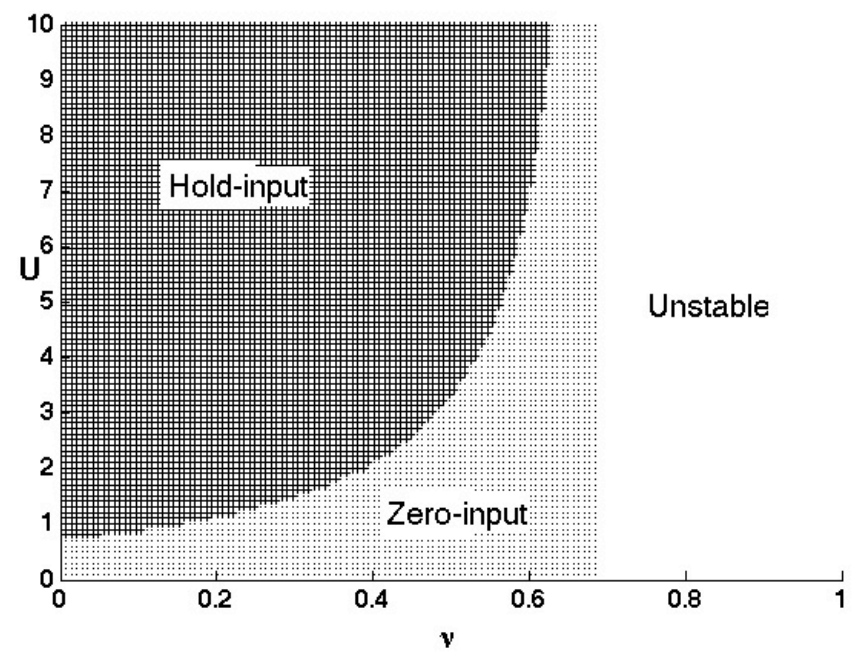

Fig. 4. Regions indicating best performing strategy in the space $(\nu, U)$ for the hold-input and the zero-input control for $A=1.2, B=1, W=1$. Stability regions for both strategies are $\nu \in\left[0, \nu_{M}\right), \nu_{M}=1 / 1.2^{2}=0.694$.

This implies that, in general, it is not possible to state whether the hold-input strategy is always better or worse than the hold-input strategy, even for simple scalar linear systems.

\section{CONCLUSION}

In this paper we studied LQ-like performance of the hold-input and zero-input strategies for control systems for which the control packets are subject to loss. These are the simplest and most commonly adopted strategies in the literature. We derived explicit expressions for computing the optimal static controller gains when control packets are lost according to a Bernoulli process. Interestingly, we showed that none of these two control schemes can be claimed to be superior to the other, even in simple scalar systems. However, the tools developed in this paper can be used to evaluate which architecture performs best once the packet loss probability and the systems parameters are known. We want to remark that although the zero-input strategy has been proposed in the literature mainly for mathematical reasons, in many situations it performs better than the hold-input strategy, thus encouraging further investigation in experimental settings and justifying its use in networked control systems. Possible future extensions of this work includes the analysis of LQG control for the hold-input strategy with dynamic output feedback, and the adoption of more realistic packet drop models.

\section{REFERENCES}

[1] J. Hespanha, P. Naghshtabrizi, and Y. Xu, "A survey of recent results in networked control systems," Proceedings of the IEEE, special issue, vol. 95, no. 1, pp. 138-162, January 2007.

[2] N. Nahi, "Optimal recursive estimation with uncertain observation," IEEE Trans. on Information Theory, vol. 15, no. 4, pp. 457-462, 1969.

[3] W. NaNacara and E. Yaz, "Linear and nonlinear estimation with uncertain observations," in American Control Conference (ACC'94), vol. 2, 1994, pp. 1429-1433.

[4] J. Nilsson, "Real-time control systems with delays," Ph.D. dissertation, Department of Automatic Control, Lund Institute of Technology, 1998.

[5] O. Costa, "Stationary filter for linear minimum mean square error estimator of discrete-time markovian jump systems," Transactions on Automatic Control, vol. 48, no. 7, pp. 1351-1356, 2002.

[6] S. Smith and P. Seiler, "Estimation with lossy measurements: jump estimators for jump systems," IEEE. Trans. Automat. Control, vol. 48, no. 12 , pp. 2163-2171, 2003.

[7] D. Giorgiev and D. Tilbury, "Packet-based control," in Proc. of the 2004 Amer. Contr. Conf., June 2004, pp. 329-336.

[8] B. Sinopoli, L. Schenato, M. Franceschetti, K. Poolla, M. Jordan, and S. Sastry, "Kalman filtering with intermittent observations," IEEE Transactions on Automatic Control, vol. 49, no. 9, pp. 1453-1464, September 2004.

[9] L. Schenato, "Kalman filtering for networked control systems with random delay and packet loss," in Conference of Mathematical Theroy of Networks and Systems (MTNS'06), Kyoto, Japan, July 2006.

[10] M. B. W. Zhang and S. Phillips, "Stability of networked control systems," IEEE Control Systems Magazine, vol. 21, no. 1, pp. 84-99, February 2001.

[11] Q. Ling and M. Lemmon, "Optimal dropout compensation in networked control systems," in IEEE conference on decision and control, Maui, HI, December 2003.

[12] N. Elia and J. Eisembeis, "Limitation of linear control over packet drop networks," in Proceedings of IEEE Conference on Decision and Control, vol. 5, Bahamas, December 2004, pp. 5152-5157.

[13] O. C. Imer, S. Yuksel, and T. Basar, "Optimal control of dynamical systems over unreliable communication links," Automatica, vol. 42, no. 9, pp. 1429-1440, September 2006.

[14] L. Schenato, B. Sinopoli, M. Franceschetti, K. Poolla, and S. Sastry, "Foundations of control and estimation over lossy networks," Proceedings of the IEEE, vol. 95, no. 1, pp. 163-187, January 2007.

[15] H. Mo and C. N. Hadjicostis, "Feedback control over packet dropping network links," in Proceedings of IEEE Mediterranean Conference on Control and Automation (MED’07), 2007, pp. 1-6.

[16] N. Elia, "Remote stabilization over fading channels," Systems and Control Letters, vol. 54, pp. 237-249, 2005.

[17] V. Gupta, S. Adlakha, B. Sinopoli, and A. Goldsmith, "Towards receding horizon networked control," Submitted to Automatica, 2007.

[18] M. Epstein, L. Shi, S. D. Cairano, and R. M. Murray, "Control over a network: Using actuation buffers and reducing transmission frequency," in 2007 European Control Conference (ECC'07), Kos, Greece, 2007.

[19] G. Chen, G. Chen, and S. Hsu, Linear Stochastic Control Systems. CRC Press, 1995

[20] O. Costa, M. Fragoso, and R. Marques, Discrete-Time Markov Jump Linear Systems (Probability and its Applications). Springer, 2004. 